\title{
Interaction of CORM-2 with hydrophobic sites: Beyond CO*
}

\author{
Elena A. Sher ${ }^{1}$, Mati Shaklai ${ }^{2}$, Nurith Shaklai ${ }^{1 \#}$ \\ ${ }^{1}$ Departments of Human Genetics \& Biochemistry, Sackler Faculty of Medicine, Tel Aviv University, Tel Aviv, Israel \\ ${ }^{2}$ Departments of Hematology, Sackler Faculty of Medicine, Tel Aviv University, Tel Aviv, Israel \\ Email: $\underline{\text { nshaklai@post.tau.ac.il }}$
}

Received 9 January 2012; revised 5 February 2012; accepted 10 February 2012

\begin{abstract}
Carbon monoxide releasing molecules (CORMs) have been recently developed for research and pharmacological purposes. A considerable amount of studies demonstrated a wide spectrum of biological activities for lipophilic CORM-2 (tricarbonyldichlororuthenium (II) dimer). It is generally accepted that the liberated gas provides the specific activities to CORMs, with a little attention paid to any possible effect of complementary core molecules. However, the versatile repertoire of actions attributed to CORM-2 is surprisingly wide for $\mathrm{CO}$, a molecule with the sole chemical activity of binding to ferrous iron in protein prosthetic groups. The study was designed to analyze CORM-2 and its core molecule ("ii"CORM) activities at a molecular level. With respect to the hydrophobic nature of the compounds, we followed their interactions with several amphipathic entities: the heme sites of hemoproteins, heme binding proteins and cell membranes. CORM-2/“ii"CORM decreased the Soret optical density of hemoglobin and myoglobin, indicating that both compounds interact with the protein amphipathic site in the heme pocket. Pre-addition of CORM-2/"ii"CORM to the apo-forms of the plasma heme binding proteins, hemopexin and albumin, partially abolished their heme binding capacity. In contrast, the compounds had no effect on the preformed heme-protein complexes. Addition of CORM-2/“i'"CORM to blood or isolated erythrocytes revealed aggregation of the cells or lysis, depending on the reagent-to-cells ratio. It was concluded that the ruthenium containing core molecule of CORM-2 may be physiologically active due to non-specific hydrophobic interactions. As each type of CORMs is expected to have a different mode of action beyond $\mathrm{CO}$ activity, their potential therapeutic uses will require clarification.
\end{abstract}

\footnotetext{
"This work was performed in partial fulfillment of the requirements for a Ph.D. degree of Elena A. Sher, Sackler Faculty of Medicine, TelAviv University, Israel.

${ }^{\#}$ Corresponding author.
}

Keywords: Carbon Monoxide; CO-Releasing Molecules; Heme; Ruthenium Complexes; Hemoproteins; Hemopexin; Albumin; Red Cell Membranes; Hydrophobic Interactions

\section{INTRODUCTION}

Many of the physiological effects attributed to hemecatabolizing enzyme Heme Oxygenase-1 (HO-1) are actually mediated by its gaseous product carbon monoxide (CO), the molecule responsible for the high affinity binding to ferrous iron $\left(\mathrm{Fe}^{\mathrm{II}}\right)$ in protein prosthetic groups. $\mathrm{HO} / \mathrm{CO}$ participates in the regulation of key physiological and pathological processes such as the resolution of inflammation, alleviation of cardiovascular disorders, as well as other conditions which have been thoroughly described in the scientific literature [1,2]. As a gas molecule, $\mathrm{CO}$ is membrane permeable, an advantage for inhaled gas administration for therapeutic aims. However, direct targeting to the desired location is limited due to $\mathrm{CO}$ high affinity binding to cytochrome oxidase and hemoglobin. To provide a bypass for the latter difficulty, a chemically diverse group of compounds with a common ability to liberate $\mathrm{CO}$, termed CO-releasing molecules (CORMs), were developed. The first to be characterized as a $\mathrm{CO}$ donor for therapeutic purposes was the transition metal carbonyl, CORM-2 (tricarbonyldichlororuthenium (II) dimer, $\left[\mathrm{Ru}(\mathrm{CO})_{3} \mathrm{Cl}_{2}\right]_{2}$ ) [3]. Over the past several years a numerous studies on the molecular, cellular, whole organ and in vivo levels demonstrated a wide spectrum of biological activities for this lipophilic molecule. Used at a broad concentration range of 10 $2000 \mu \mathrm{M}$, it was shown to participate in a variety of essential physiological processes: ion channel regulation [4,5], coagulation [6], angiogenesis [7], etc. Studies in the literature relied on a consensus considering CORM-2 activity to be the outcome of the released $\mathrm{CO}$ either employing DMSO alone (vehicle) as well as the inactive control $\mathrm{RuCl}_{3}$ or the water soluble $\mathrm{Ru}^{\mathrm{II}}$ complex, $\mathrm{RuCl}_{2}(\mathrm{DMSO})_{4}[8]$ or the CORM-2 residual following 
CO release, termed inactive CORM, iCORM [5].

Although it is generally accepted that the released CO provides the specific activity to CORMs, the versatile repertoire of actions attributed so far to CORM-2 is surprisingly wide for a single molecule with narrow chemical activity like CO. Indeed, at least two studies reported that CORM-2 exerts different activities independent of its ability to release CO $[4,5]$. Despite numerous reports supporting the potential of CORMs, in particular CORM-2, in development of therapeutic strategies, molecular-level elucidation is scarce. The current study focused on analyzing a molecular activity of CORM-2 which, a priori, can be an outcome of the released $\mathrm{CO}$ or the core molecule, to be referred to as "i"CORM. With respect to the hydrophobic nature of CORM-2, we chose to analyze its interactions with well-known amphipathic entities, like heme sites of hemoproteins, heme binding proteins as well as cell membranes.

\section{MATERIALS AND METHODS}

\subsection{Materials}

Tricarbonyldichlororuthenium (II) dimer (CORM-2), albumin (from human serum, HSA), Hemin (from bovine red cells, H), myoglobin (equine, $\mathrm{Mb}$ ), cytochrome $\mathrm{C}$ (from bovine heart, cytC), phenylmethylsulfonyl fluoride (PMSF) and dithionite were all from Sigma-Aldrich. Dimethylsulfoxide (DMSO) was purchased from Merck, Darmstadt, Germany. PD-10 desalting columns were purchased from GE Healthcare UK Ltd. S-Sepharose column was obtained from Pharmacia, Uppsala, Sweden. CM-52 and DE-52 celluloses were obtained from Whatman International, Maidstone, England. Ferricyanide was purchased from Agan Chemical Manufacturers Ltd., Israel. Human plasma was supplied by the Israeli central blood bank. Nitrogen gas $\left(\mathrm{N}_{2}, 99.9 \%\right)$ was supplied by Israel Oxygen Centre.

\subsection{Preparation of Proteins}

Hemoglobin: human hemoglobin was purified from human red blood cell lysate by ion-exchange chromatography using CM-52 cellulose followed by extensive dialysis against PBS, pH 7.4 in oxygen free atmosphere [9]. It was spectrophotometrically verified as oxyHb and stored at $-70^{\circ} \mathrm{C}$ in oxygen free atmosphere. Ferric hemoglobin (metHb) was prepared from the oxy form by oxidation with potassium ferricyanide [9] followed by reagent removal on a PD-10 desalting column and used on the same day of preparation [10].

Myoglobin: commercially available metmyoglobin (metMb) was dissolved in PBS (pH 7.4) and free heme removed by mixing the stock solution with an equal volume of PBS-equilibrated DE-52 ion exchange resin followed by centrifugation [9]. Oxymyoglobin (oxyMb) was prepared from the met form by reduction with excess of dithionite followed by reagent removal using PD-10 desalting column. Columns were routinely equilibrated with $\mathrm{N}_{2}$ saturated buffer and the developing buffer was $\mathrm{N}_{2}$ saturated as well.

Hemoproteins (expressed as heme equivalents) were quantified spectrophotometrically using the following extinction coefficients: $\varepsilon_{414 \mathrm{~nm}}=125 \mathrm{mM}^{-1} \cdot \mathrm{cm}^{-1}$ in $\mathrm{pH}$ 7.4 for oxyHb; $\varepsilon_{405 \mathrm{~nm}}=179 \mathrm{mM}^{-1} \cdot \mathrm{cm}^{-1}$ in $\mathrm{pH} 6.3$ for metHb; $\varepsilon_{418 \mathrm{~nm}}=120 \mathrm{mM}^{-1} \cdot \mathrm{cm}^{-1}$ in $\mathrm{pH} 7.4$ for oxyMb and $\varepsilon_{408 \mathrm{~nm}}=188 \mathrm{mM}^{-1} \cdot \mathrm{cm}^{-1}$ in $\mathrm{pH} 6.3$ for metMb [9].

Human hemopexin $(\mathrm{Hx})$ : Hx was isolated from fresh frozen plasma of healthy donors according to published method [11,12] modified by using only SP-Sepharose fast flow column, which yielded sufficiently purified protein as verified by SDS-PAGE, lyophilized and stored at $-20^{\circ} \mathrm{C}$. The concentration of apoHx was determined by spectrophotometric titration with free hemin based on heme-hemopexin specific absorption [12].

\subsection{CORM-2/“ii”CORM Interactions with Proteins}

A stock solution of CORM-2 was prepared in DMSO. Upon solubilization the dimer dissociates to monomers with at least one $\mathrm{CO}$ molecule liberated immediately and DMSO attaching to form intermediates $\left(\mathrm{Ru}(\mathrm{CO})_{3} \mathrm{Cl}_{2}\right.$ (DMSO) \& $\left.\mathrm{Ru}(\mathrm{CO})_{2} \mathrm{Cl}_{2}(\mathrm{DMSO})_{2}\right)$ [3]. The stock solution was further diluted to the desired concentration in PBS pH 7.4, reaching 0.5\% - 1.25\% DMSO which was identical in both control and experimental mixtures. To avoid time dependent spectral contribution from water phase-dissolved CORM-2 at each experimental time point, absorbance from a CORM-2 control mixture was subtracted (see Figure 1(a)) and hence throughout the study difference spectra only are presented.

"i"CORM (iCORM-2) was prepared by allowing CORM-2 stock solution to equilibrate with air for at least 72 hours at RT. Since CORM-2 is a dimer while " $\mathrm{i}$ "CORM is a monomer in solution [3], for consistency concentrations were expressed throughout this study in dimer units. Mixing time was up to $1 \mathrm{~min}$. The proteins were incubated with desired reagents in PBS $\mathrm{pH} 7.4$ at RT.

Heme-protein complexes, H-Hx and H-HSA, were prepared by mixing a small molar excess of apo-protein with free heme stock in DMSO.

\subsection{CORM-2/“ii”CORM Interactions with Red Blood Cells}

Blood was collected from healthy human donors into EDTA containing vacuum tubes by venipuncture and used for experiments on the same day of collection.

Interaction with whole blood: To avoid direct red 

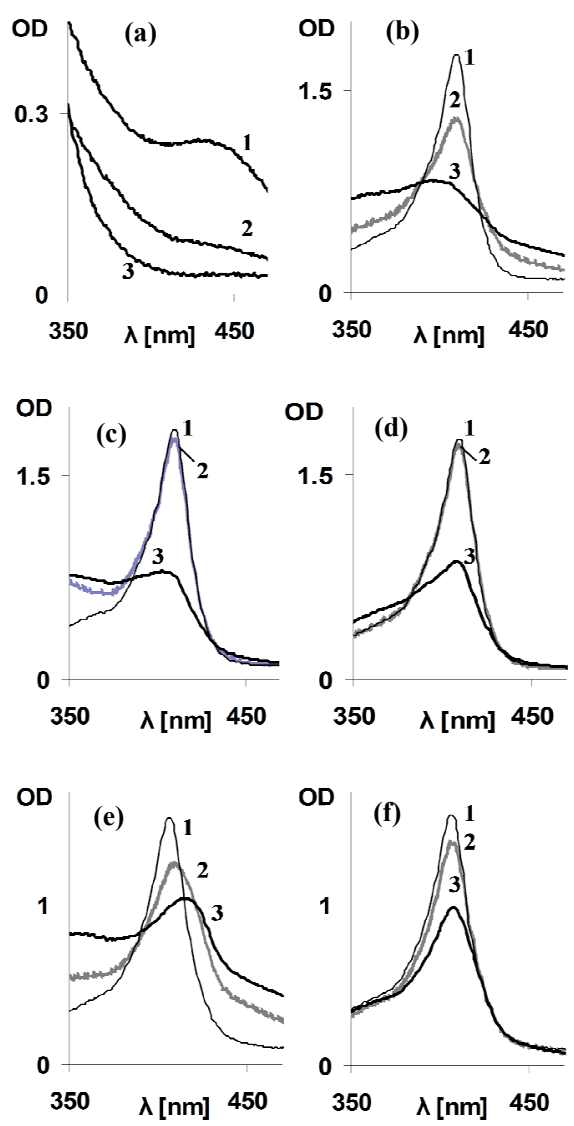

Figure 1. Effect of CORM-2/“i”CORM on Soret spectra of metRH. (a) Time dependent spectral changes of solubilized CORM-2; Line 1: CORM-2 $(1000 \mu \mathrm{M})$ at mixing time (CORM-2 dissolved in DMSO and following $10 \mathrm{~min}$ diluted into PBS); line 2: As 1 following 2 hours of incubation in RT; line 3: "i"CORM (1000 $\mu \mathrm{M}$ per dimer) prepared by allowing CORM-2 stock solution $(200 \mathrm{mM}$ in DMSO) to incubate at RT for at least 72 hours and then diluted into PBS. (b)-(d) Effect of CORM-2/ "i"CORM on Soret spectrum of metMb; (b) High CORM-2 concentration $(1000 \mu \mathrm{M})$; (c) Low CORM-2 $(100 \mu \mathrm{M})$; (d) "i"CORM-2 $(1000 \mu \mathrm{M}$ per dimer) Lines 1 (thin): metMb alone; Lines 2 (gray) as 1+CORM-2/“i”CORM at mixing time; Lines 3 (heavy): 2, following 2 hours of incubation in RT. MetMb concentration $-10 \mu \mathrm{M}$. (e)-(f) Effect of CORM-2/"i"CORM on Soret spectrum of metHb; (e) CORM-2 $(1000 \mu \mathrm{M})$; (f) iCORM-2 $(1000 \mu \mathrm{M}$ per dimer). Lines 1 (thin): metHb alone; Lines 2 (gray): metHb + CORM-2/“i”"CORM at mixing time; Lines 3 (heavy): 2, following 2 hours of incubation in RT. MetHb concentration $-10 \mu \mathrm{M}$.

blood cells (RBC) interactions with concentrated DMSO, stock solutions of CORM-2/“i"CORM or pure DMSO control were initially diluted 1:10 with PBS and these solutions were added to the fresh blood to yield $1000 \mu \mathrm{M}$ CORM concentration.

Interaction with isolated $\mathrm{RBC}$ suspension: $\mathrm{RBC}$ were isolated and washed in PBS. Concentrated cells were first diluted 1:500 into PBS pH 7.4. This stock was further diluted 1:4 in CORM-2/DMSO containing PBS for experimental mixtures or in DMSO containing DDW for the maximal hemolysis measurement. Experimental mixtures were incubated at RT with gentle shaking. Light absorption spectra of the supernatants followed $10 \mathrm{~min}$ utes centrifugation at $1000 \times \mathrm{g}$ were measured. Optical density (OD) at Soret maxima served for the estimation of hemolysis level.

\subsection{Spectrophotometry}

Spectral characteristics of stock solutions and reaction mixtures were determined by scanning the light absorption spectra at visible wavelength range $(350-700 \mathrm{~nm})$ using a Contron-UVIKON $\mathrm{XL}_{\mathrm{XL}}$ or GBC UV/VIS 920 spectrophotometers.

\section{RESULTS AND DISCUSSION}

\subsection{Effect of CORM-2 on Ferric Forms of Hemoproteins}

Respiratory hemoproteins $(\mathrm{RH})$, myoglobin $(\mathrm{Mb})$ and hemoglobin $(\mathrm{Hb})$ contains well established amphipathic heme sites. The heme is attached to the globin non-covalently, yet with extremely high affinity and its typical Soret light absorption allows monitoring site alterations [9]. The effect of CORM-2 was initially followed on ferric $\left(\mathrm{Fe}^{\mathrm{III}}\right.$, met) forms of $\mathrm{RH}$ which do not bind $\mathrm{CO}$. Addition of CORM-2 at a relatively high concentration of $1000 \mu \mathrm{M}$ resulted in a considerable decrease of metMb Soret absorption at mixing time and the changes were practically terminated following two hours (Figure 1(b)). Increased optical density (OD) at $470 \mathrm{~nm}$, the wavelength lacking specific $\mathrm{Mb}$ absorption, indicated time dependent contributions from protein conformation and/or light scattering aggregates. Incubation of metMb with $1000 \mu \mathrm{M}$ of "i"CORM resulted in decreased Soret absorption, although with slower rate than CORM-2, as indicated by lack of spectral changes within mixing time (Figure 1(c)), and lesser light scatter. Lower CORM-2 concentration $(100 \mu \mathrm{M})$ resulted in Soret decrease as well, but at a slower rate (Figure 1(d)). The CORM2/ "i"CORM effects on RH light absorption are unexplained by any known $\mathrm{CO}$ activity. As solubilized CORM-2 has a small, time-dependent absorption in the Soret region shown in Figure 1(a), throughout the study all results were corrected for this contribution. In presence of $\mathrm{RuCl}_{3}(250 \mu \mathrm{M}$, the limit of its solubility) the Soret peak of metMb was unchanged (data not shown).

To clarify if the above effects are $\mathrm{Mb}$ specific, similar experiments were carried out using metHb. Addition of $1000 \mu \mathrm{M}$ CORM-2 to metHb resulted in the following spectral changes: a fast (within mixing time) Soret re- 
duction, red shift and light scatter elevation, all reaching a steady state within 2 hours (Figure 1(e), spectra $2 \& 3$ ). "i"CORM caused Soret reduction as well, but with slower kinetics (Figure 1(f)). Moreover, unlike the case of CORM-2, neither red shift nor any light scatter elevation were observed following "i" CORM addition.

The RH Soret decrease by addition of CORM-2/ "i"CORM can be explained by expulsion of heme from the globin and/or its disintegration. These changes are the outcome of hydrophobic nature of CORM-2 and intermediates in the process of $\mathrm{CO}$ release reaching finally "i"CORM, all potentially competing with heme for the protein site. Higher activity of CORM-2 indicated that the intermediates formed upon $\mathrm{CO}$ release (see methods), may kinetically attach to the heme site via hydrophobic interactions prior to DMSO binding [3]. The slight red shift of $\mathrm{Hb}$ Soret, observed in the case of CORM-2 only, may result from partial reduction of the heme iron $\left(\mathrm{Fe}^{\mathrm{III}} \rightarrow \mathrm{Fe}^{\mathrm{II}}\right)$ and carboxyHb $(\mathrm{COHb})$ formation, which can occur by electron transfer from a neighboring residue like cysteine, in presence of $\mathrm{CO}$ [13].

To assess if CORM-2 has similar effects on other hemoproteins, $1000 \mu \mathrm{M}$ CORM-2/“i"CORM were added to cytochrome $\mathrm{C}$ in which the heme is covalently bound and surface unexposed [14]. No effect on the typical Soret spectrum of this hemoprotein was observed following incubation of several hours (data not shown). These findings supported the involvement of hydrophobic interactions in the effects of CORM-2 on RH.

\subsection{Effect of CORM-2 on Ferrous Forms of Respiratory Hemoproteins}

In ferrous ( $\mathrm{Fe}^{\mathrm{II}}$, oxy) $\mathrm{Hb}$ the heme-to-globin bond is much stronger as compared to the ferric forms [15], and is even stronger in $\mathrm{COHb}$ as compared to oxy form [9]. Thus it was important to find out if CORM-2 can compete with ferrous heme. Addition of $1000 \mu \mathrm{M}$ CORM-2 to oxyHb, the concentration at which a fast reaction with met form observed, resulted in an increased absorption and a significant red shift of the Soret peak within mixing time (Figure 2). The latter alterations point at transformation of oxyHb (414 nm peak) to $\mathrm{COHb}(419 \mathrm{~nm}$ peak). However, while the theoretically, the complete transformation should yield a 1.52 -fold increase in OD [9], only a 1.16-fold increase was observed (Figure 2). Moreover, following 2 hours, the absorption of the mixture further decreased while the Soret features remained typical for $\mathrm{COHb}$. These data indicate that despite the carboxy-complex formation, a state which strengthens the heme-globin interaction, CORM-2 could still compete with the heme on the site [9].

Identical experiments were performed using oxyMb. Addition of $1000 \mu \mathrm{M}$ CORM-2 caused a slight red shift
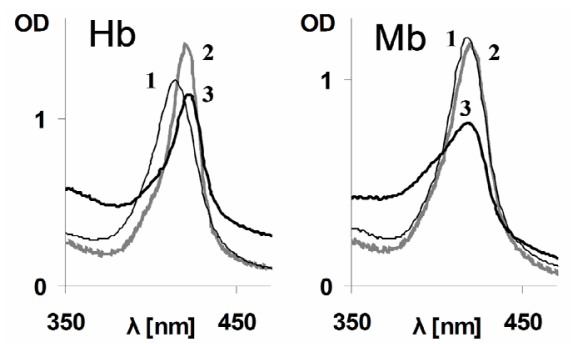

Figure 2. Effect of CORM-2 on Soret spectra of oxyRH. Lines 1 (thin): oxyhb/Mb alone; Lines 2 (gray): $\mathrm{Hb} / \mathrm{Mb}+\mathrm{CORM}-2(1000 \mu \mathrm{M})$ at mixing time; Lines 3 (heavy): 2, following 2 hours of incubation in RT. RH concentration $-10 \mu \mathrm{M}$.

within the mixing time, indicating only negligible transformation of oxyMb into its carboxy form, although over time the Soret absorption decreased (Figure 2). The difference between $\mathrm{Hb}$ and $\mathrm{Mb}$ appears to be related to the fact that affinity of $\mathrm{CO}$ for $\mathrm{Mb}\left(\mathrm{Fe}^{\mathrm{II}}\right)$ is not as high and thus conversion of oxy to carboxy form is less efficient than in the case of $\mathrm{Hb}$ [9].

To evaluate whether the interaction of CORM-2/ " $i$ "CORM with hydrophobic sites in proteins is a more general phenomenon, similar studies were performed with plasma proteins known to contain amphipathic sites for heme and/or other small molecules.

\subsection{Effect of CORM-2 and "ip"CORM on Plasma Heme Binding Proteins}

Hemopexin (Hx) has a uniquely high affinity $\left(>10^{13}\right)$ for the ferric heme. Its single specific heme binding site is composed of eight hydrophobic residues in addition to coordination bonds [16]. Formation of the heme-hemopexin complex $(\mathrm{H}-\mathrm{Hx})$ results in an immediate appearance of the typical $414 \mathrm{~nm}$ Soret peak (Figure 3 (upper), spectrum 2), which known not to be affected by CO gas [12]. Addition of $1000 \mu \mathrm{M}$ CORM-2/“i" $\mathrm{i}$ "CORM to H-Hx did not affect the Soret absorption band. Nonetheless, preincubation of CORM-2/“i"CORM with apoHx followed by heme addition resulted in formation of a much smaller Soret absorption (Figure 3 (upper), spectrum 3).

That H-Hx was not affected by CORM-2/“i"CORM can be explained by the fact that heme binding to apoHx is followed by global conformational changes, shielding the heme from the water phase [17]. In the case of heme interaction with the CORM-2/"i"CORM preincubated apoHx, the residual Soret peak at $414 \mathrm{~nm}$ is interpreted as competitive binding of the above compounds to the Hx heme binding site. An additional and/or alternative explanation is the possibility that CORM-2/“i" $\mathrm{l}$ "CORM binding, like heme, is followed by protein conformational changes resulting in inaccessibility of the site to any ligand. 


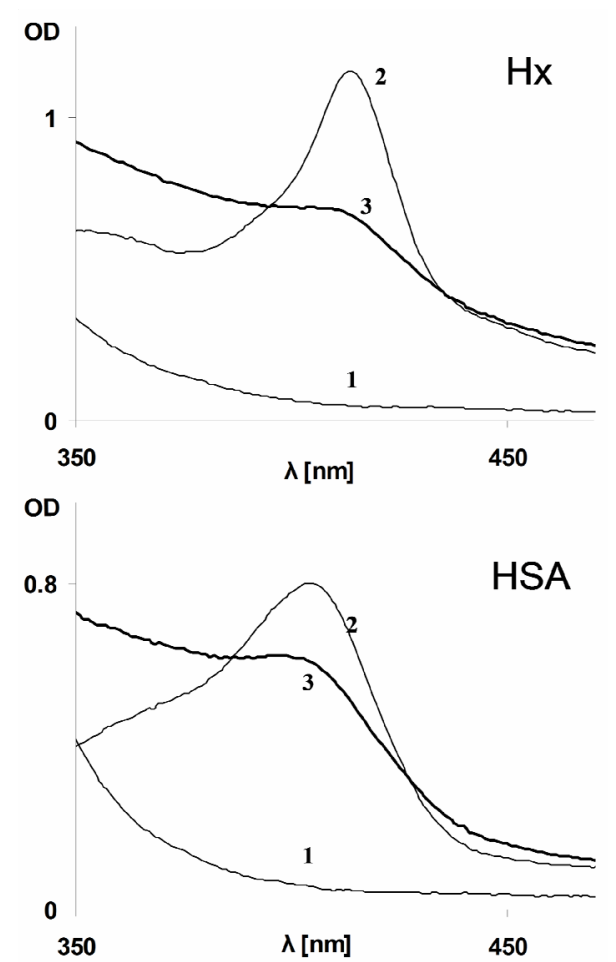

Figure 3. Effect of "i"CORM-2 on hemin binding to plasma proteins. Upper: $\mathrm{Hx}(12.5 \mu \mathrm{M}$, by activity—see methods); Lower: HSA $(12 \mu \mathrm{M})$. Lines 1: "i"CORM alone (1000 $\mu \mathrm{M}$ per dimer); Lines 2: Typical H-Hx/H-HSA complex formed by $10 \mu \mathrm{M}$ of heme in PBS containing no "i"CORM; Lines 3: A mixture of hemin with apoHx/HSA pre-incubated for 5 minutes in presence of "i”CORM (1000 $\mu \mathrm{M}$ per dimmer).

It was of interest to determine if CORM-2/“i" $\mathrm{i}$ "CORM can interact with human serum albumin (HSA), known to have a single high affinity heme binding site as well as several non-specific amphipathic sites $[18,19]$. Experiments similar to those carried out with $\mathrm{Hx}$ were repeated employing HSA, using hemin concentration allowing only its high affinity site to be potentially occupied. In this case as well, addition of CORM-2/“i" $\mathrm{i}$ "CORM did not affect the spectrum of the preformed heme-albumin complex (H-HSA). Addition of heme following pre-incubation of HSA with CORM-2/"i"CORM resulted in similar Soret spectrum but with decreased OD (Figure 3 (lower), spectrum 3).

Thus, heme binding capacity of both Hx and HSA was lost by CORM-2/"i"CORM treatment but the leftover heme binding sites were unchanged as identified by Soret. To estimate the fraction of remaining heme sites, $\triangle \mathrm{OD}$ of the Soret peak (414 nm for Hx and $404 \mathrm{~nm}$ for HSA) and isosbestic wavelength at the red part of the spectrum (where no contributions from the Soret band are available) was calculated. These calculations indicated that $62 \%$ of $\mathrm{Hx}$ and $37 \%$ of HSA still bound heme in presence of CORMs.

That in comparison to Hx, albumin lost less of its heme binding capacity, despite weaker heme affinity, can be explained by CORMs hydrophobicity. CORMs appears to be less available for the high affinity heme binding site as it is mostly bound to the broad range of non specific sites of HSA, while in Hx it only competes for the single heme-site.

Since CORMs are designed to be applicable for clinical use it should be noted that binding of CORM/ "i"CORM to Hx may prevent its vital physiological activeties such as clearance of differentiating erythroid progenitors membrane from accumulated hemin [20]. In the case of albumin, the major blood protein which binds non specifically low molecular weight amphipathic drugs [21], administration of CORM might alter their pharmacokinetics.

\subsection{CORM-2 Effects on Cell Membranes}

The hydrophobic nature of CORM-2/“i”"CORM binding implies possible general nonspecific association with hydrophobic surfaces of proteins as well as cell membranes. Thus we further analyzed CORM-2/“i" $\mathrm{i}$ CORM effect on whole blood.

CORM-2/“i"CORM $(1000 \mu \mathrm{M})$ or DMSO alone as control were added to fresh whole blood samples (see methods). Representative results are demonstrated in Figure 4(a) micrographs. As can be seen, aggregates composed of few red cells were formed by addition of CORM-2/“i”'CORM but were not observed in presence of DMSO alone. Previous literature reported red blood cells (RBC) aggregation in blood smears from patients that have received hydrophobic drugs [22]. Thus the aggregates formation in the presence of CORM-2/“i" CORM is consistent with their hydrophobic nature.

To shed further light on the RBC membrane changes which might have caused aggregation, diluted isolated $\mathrm{RBCs}$ which yield the same $\mathrm{CORM} / \mathrm{Hb}$ ratio as in the first section of the study were incubated with $1000 \mu \mathrm{M}$ CORM-2. Large brown non-resuspendable clumps of precipitate immediately formed leaving no soluble free $\mathrm{Hb}$ in the buffer. Therefore we lowered the concentration of CORM-2 to $100 \mu \mathrm{M}$ which resulted in time dependent hemolysis. Quantitation of the hemolysis was estimated by cell-free oxyHb concentration following 6 hours of incubation (as oxidation/denaturation developed later). The results are shown in Figure 4(b). As can be seen, DMSO alone produced $13 \%$ hemolysis while $100 \mu \mathrm{M}$ of CORM-2 produced a significant increased hemolysis. These results could not be attributed to stabilizing the ferrous heme-iron by the $\mathrm{CO}$ liberated from the donor molecule [15]. The hemolysis can be explained by the hydrophobic nature of CORM-2 and all of its intermediates during $\mathrm{CO}$ release, allowing their intercalation within 
(a)

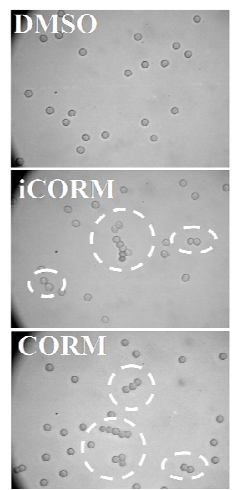

(b)

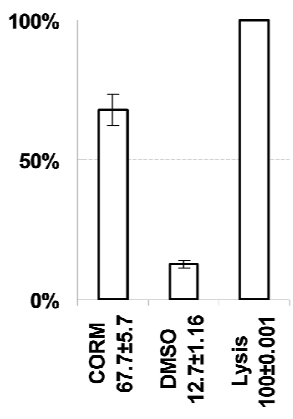

Figure 4. Effect of CORM-2/“i”"CORM on red cell membranes. (a) CORM-2/“i"CORM induced red cells aggregation in whole blood. Upper: fresh blood + DMSO 0.5\%; Middle: As upper plus CORM-2 (1000 $\mu \mathrm{M})$; Lower: As upper plus "i"CORM (1000 $\mu \mathrm{M}$ per dimer). (2 $\mu \mathrm{l}$ of blood exposed to the reagent was mixed with $1.5 \mathrm{ml} \mathrm{PBS}$ and placed in hemacytometer for microscopic observation.) (b) CORM-2 induced hemolysis in RBC suspension. Acellular oxyHb relative concentrations in buffer following $\mathrm{RBC}$ suspension incubation $(\mathrm{t}=6 \mathrm{~h})$ in presence of CORM-2 $(100 \mu \mathrm{M})$ or DMSO alone (control) presented as a percent of maximal value reached by hypotonic hemolysis. Data from 2 donors and 3 repetitions in each.

cell membrane and its deterioration, a general phenomenon of hydrophobic detergents.

Finally, we have shown in the above study that CORM-2 provides non-specific, hydrophobic interactions with a variety of amphipathic surfaces. Such effects appear to be unrelated to CO activity. This observation indicates that during the gas release the intermediate states of hydrophobic complementary core molecules afford this activity, even though the final "i"CORM is less active.

This phenomenon appears to cause a misconception in many studies that relate CORM activity solely to the released CO. For example, it was suggested that the molecular basis of CORM-2 activity as procoagulant is based on CO binding to a fibrin associated heme [23], while the coagulant activity of fibrin depends on transformation of hydrophilic fibrinogen to the hydrophobic fibrin [24]. The above observations combined with those presented here, suggest that ruthenium-containing core molecules confer to CORM-2 its procoagulant properties.

It should be emphasized that the current study findings are not antagonistic to the pharmacological use of CORMs. In contrast, the outcome of the study suggests that both $\mathrm{CO}$ and the complementary part of CORM-2 may be physiologically active. These two parts demonstrate completely different activities: $\mathrm{CO}$ interacts spe- cifically with divalent transition metals, while the core molecule acts via non-specific hydrophobic interactions. While the same $\mathrm{CO}$ gas is liberated from all types of CORMs and only the kinetics of its release is evaluated, the chemical nature of the complementary core molecules may by hydrophobic, amphipathic or charged and therefore, each should be studied for positive/negative side effects independently.

\section{ACKNOWLEDGEMENTS}

This study was supported by hematological research fund in memory of Kravitz family (to Mati Shaklai). The authors would like to thank Y. Goldman for valuable editorial assistance.

\section{REFERENCES}

[1] Ryter, S.W. and Choi, A.M. (2009) Heme oxygenase1/carbon monoxide: From metabolism to molecular therapy. American Journal of Respiratory Cell and Molecular Biology, 41, 251-260. doi:10.1165/rcmb.2009-0170TR

[2] Gozzelino, R., Jeney, V. and Soares, M.P. (2010) Mechanisms of cell protection by heme oxygenase-1. Annual Review of Pharmacology and Toxicology, 50, 323-354. doi:10.1146/annurev.pharmtox.010909.105600

[3] Motterlini, R., Clark, J.E., Foresti, R., et al. (2002) Carbon monoxide-releasing molecules: Characterization of biochemical and vascular activities. Circulation Research, 90, 17-24. doi:10.1161/hh0202.104530

[4] Dong, D.L., Chen, C., Huang, W., et al. (2008) Tricarbonyldichlororuthenium (II) dimer (CORM2) activates non-selective cation current in human endothelial cells independently of carbon monoxide releasing. European Journal of Pharmacology, 590, 99-104.

doi:10.1016/j.ejphar.2008.05.042

[5] Wilkinson, W.J. and Kemp, P.J. (2011) The carbon monoxide donor, CORM-2, is an antagonist of ATP-gated, human P2X4 receptors. Purinergic Signalling, 7, 57-64. doi:10.1007/s11302-010-9213-8

[6] Nielsen, V.G., Kirklin, J.K. and George, J.F. (2009) Carbon monoxide-releasing molecule-2 increases the velocity of thrombus growth and strength in human plasma. Blood Coagulation \& Fibrinolysis, 20, 377-380. doi:10.1097/MBC.0b013e32832ca3a3

[7] Deshane, J., Chen, S., Caballero, S., et al. (2007) Stromal cell-derived factor 1 promotes angiogenesis via a heme oxygenase 1-dependent mechanism. The Journal of Experimental Medicine, 204, 605-618. doi:10.1084/jem.20061609

[8] Motterlini, R. and Otterbein, L.E. (2010) The therapeutic potential of carbon monoxide. Nature Reviews Drug Discovery, 9, 728-743. doi:10.1038/nrd3228

[9] Antonini, M.E. and Brunori, M. (1971) The derivatives of ferric hemoglobin and myoglobin. North-Holland Publishing Company, Amsterdam.

[10] Ueno, R., Shimizu, T., Kondo, K., et al. (1982) Activation mechanism of prostaglandin endoperoxide synthetase by 
hemoproteins. The Journal of Biological Chemistry, 257, 5584-5588.

[11] Hrkal, Z., Cabart, P. and Kalousek, I. (1992) Isolation of human haemopexin in apo-form by chromatography on S-Sepharose Fast Flow and Blue Sepharose CL-6B. Biomedical Chromatography, 6, 212-214. doi:10.1002/bmc. 1130060412

[12] Tsemakhovitch, V.A., Bamm, V.V. and Shaklai, N. (2005) Vascular damage by unstable hemoglobins: The role of heme-depleted globin. Archives of Biochemistry and Biophysics, 436, 307-315. doi:10.1016/j.abb.2005.02.006

[13] Hirota, S., Azuma, K., Fukuba, M., et al. (2005) Heme reduction by intramolecular electron transfer in cysteine mutant myoglobin under carbon monoxide atmosphere. Biochemistry, 44, 10322-10327. doi:10.1021/bi0507581

[14] Prabhu, N.P., Kumar, R. and Bhuyan, A.K. (2004) Folding barrier in horse cytochrome c: Support for a classical folding pathway. Journal of Molecular Biology, 337, 195208. doi: $10.1016 /$ j.jmb.2004.01.016

[15] Bunn, H.F. and Jandl, J.H. (1968) Exchange of heme among hemoglobins and between hemoglobin and albumin. The Journal of Biological Chemistry, 243, 465-475.

[16] Liem, H.H., Spector, J.I., Conway, T.P., et al. (1975) Effect of hemoglobin and hematin on plasma clearance of hemopexin, photo-inactivated hemopexin and albumin. Proceedings of the Society for Experimental Biology, 148, 519-522.

[17] Paoli, M., Anderson, B.F., Baker, H.M., et al. (1999) Crystal structure of hemopexin reveals a novel high-affinity heme site formed between two beta-pro-peller do- mains. Nature Structural \& Molecular Biology, 6, 926931. doi:10.1038/13294

[18] Beaven, G.H., Chen, S.H., d'Albis, A. and Gratzer, W.B. (1974) A spectroscopic study of the haemin-human-serum-albumin system. European Journal of Biochemistry, 41, 539-546. doi:10.1111/j.1432-1033.1974.tb03295.x

[19] Zunszain, P.A., Ghuman, J., Komatsu, T., et al. (2003) Crystal structural analysis of human serum albumin complexed with hemin and fatty acid. BMC Structural Biology, 3, 6. doi:10.1186/1472-6807-3-6

[20] Yang, Z., Philips, J.D., Doty, R.T., et al. (2010) Kinetics and specificity of feline leukemia virus subgroup $\mathrm{C}$ receptor (FLVCR) export function and its dependence on hemopexin. The Journal of Biological Chemistry, 285, 28874-28882. doi:10.1074/jbc.M110.119131

[21] Baroni, S., Mattu, M., Vannini, A., et al. (2001) Effect of ibuprofen and warfarin on the allosteric properties of haem-human serum albumin. A spectroscopic study. European Journal of Biochemistry, 268, 6214-6220. doi:10.1046/j.0014-2956.2001.02569.x

[22] Valerie, N.G. (2009) Chemical-associated artifacts. Blood, 113, 4487.

[23] Nielsen, V.G., Cohen, J.B., Malayaman, S.N., et al. (2011) Fibrinogen is a heme-associated, carbon monoxide sensing molecule: A preliminary report. Blood Coagulation \& Fibrinolysis, 22, 443-447. doi: 10.1097/MBC.0b013e328345c069

[24] van Oss, C.J. (1990) Surface properties of fibrinogen and fibrin. Journal of Protein Chemistry, 9, 487-491. doi: 10.1007/BF01024625 